Itinéraires Itinéraires

Littérature, textes, cultures

2009-2 | 2009

Caraïbe et océan Indien

\title{
Édouard Glissant, avec Alexandre Leupin, Les Entretiens de Baton Rouge
}

Stéphane Hoarau

\section{(2) OpenEdition}

1 Journals

Édition électronique

URL : http://journals.openedition.org/itineraires/228

DOI : 10.4000/itineraires.228

ISSN : 2427-920X

Éditeur

Pléiade

\section{Édition imprimée}

Date de publication : 1 juillet 2009

Pagination : 192-197

ISBN : 978-2-296-09639-4

ISSN : $2100-1340$

\section{Référence électronique}

Stéphane Hoarau, «Édouard Glissant, avec Alexandre Leupin, Les Entretiens de Baton Rouge », Itinéraires [En ligne], 2009-2 | 2009, mis en ligne le 27 mai 2014, consulté le 22 septembre 2020. URL http://journals.openedition.org/itineraires/228 ; DOI : https://doi.org/10.4000/itineraires.228

Ce document a été généré automatiquement le 22 septembre 2020.

\section{cc) (†) $\odot$}

Itinéraires est mis à disposition selon les termes de la licence Creative Commons Attribution - Pas d'Utilisation Commerciale - Pas de Modification 4.0 International. 


\title{
Édouard Glissant, avec Alexandre Leupin, Les Entretiens de Baton Rouge
}

\author{
Stéphane Hoarau
}

\section{RÉFÉRENCE}

Édouard Glissant, avec Alexandre Leupin, Les Entretiens de Baton Rouge, Paris, Gallimard, 2008

1 Au moment où ont lieu les entretiens de Baton Rouge (entre 1990 et 1991), Édouard Glissant vient d'être nommé "Professeur Distingué des Universités » à l'université d'État de la Louisiane (LSU). Il habite alors Baton Rouge et c'est dans cet espace francophone qu'il rencontre le médiéviste Alexandre Leupin. Le Graal et la littérature et Barbarolexis, Medieval Literature and Sexuality, les deux premières publications de ce dernier (respectivement en 1983 et 1989) proposaient d'étudier des textes du Moyen Âge par le biais de méthodes critiques contemporaines. Ces hommes, tous deux francophones (l'un, Français originaire de la Martinique, l'autre, Suisse), se proposent alors d'entrer en relation, d'échanger, au cours d'entretiens, leurs points de vue - selon leurs connaissances et leurs approches respectives de la littérature. Il en résulte une somme d'entrevues qui ne sera pas immédiatement publiée. Il faudra attendre 2008 pour qu'une version définitive de ces entretiens soit éditée. Les Entretiens de Baton Rouge raconte cette rencontre, sur un continent qui n'est pas l'Europe. C'est la mesure de la distance prise qui est à la source du projet: discourir sur notre contemporanéité en s'appuyant sur des expériences passées, et plus précisément, sur un moment de l'histoire occidentale qui a conditionné la trajectoire de sociétés, et qui a façonné, entre autres, notre perception universalisante du monde. Les deux hommes rappellent que le Moyen Âge est une période paradoxale qui a vu à la fois éclore les bourgeons de langues, de cultures, de nations et de conceptions identitaires et qui, dans un même temps, a vu flétrir une diversité naissante. Se heurtent alors deux entités imaginaires et cognitives: la "pensée du système", intransigeante et centrée, et la "pensée de l'hérésie », transversale et « excentrée » (28). Il en résulte une somme d'interrogations. 
L'une d'entre elles est la suivante : «Que peut-être une pensée excentrée? » La réponse suit :

Une pensée excentrée, c'est une pensée qui questionne la légitimité de l'appropriation, celle de l'extension et de l'expansion, une pensée qui, informant la notion de centre et périphérie, contribue à constituer toutes les périphéries en centres et tous les centres en périphéries d'autre chose. Et qui par conséquent amène à constituer le relatif dans la Relation. (129)

Sont réexposés des thèmes chers à Glissant présents dans toutes ses productions, avant comme après 1991 : le centre, les périphéries, la Relation et, de fait, la reconnaissance d'une diversité à même de faire vivre les jeux de relations.

Mais, croyons-nous naïvement, rappelle Édouard Glissant, l'histoire est « un processus qui part d'un endroit, qui dispose d'un moteur et qui va vers un autre endroit » (42). Il convient de dissiper ce malentendu selon lequel il y aurait eu un mouvement linéaire et chronologique qui aurait fait passer l'Occident, dans sa seule relation à lui-même, du Moyen Âge à la Renaissance, aux Lumières, et à l'histoire des traites, des esclavages, des colonisations puis des décolonisations. L'histoire contemporaine, telle que nous la concevons trop souvent, est une succession ordonnée d'événements ayant pour but ultime et absolu (au singulier donc) la modernité. L'évolution de l'Occident est ordonnée, structurée, en elle-même, par elle-même, pour elle-même. Nous ignorons généralement les apports extérieurs. Or l'histoire n'est peut-être pas un unique fil qui se déroule, mais une maille, cousue d'une multitude de fils.

3 L'universel est ce qui, dans le texte, est mis en débat. Si je choisis de restituer ces mots échangés entre Édouard Glissant et Alexandre Leupin, je risque de me perdre, et de perdre ma propre langue dans le sillon des leurs (chacun des deux intervenants ayant la sienne propre). Le projet qui prend corps à la lecture de ce livre : non pas rapporter ou retranscrire donc, mais comprendre comment pensées et concepts énoncés selon ces modalités, travaillent en effets ma perception d'une œuvre dans un paysage, jusqu'à renverser, en effets toujours, la perception de mon propre paysage culturel et imaginaire.

4 Poursuivant ma lecture des Entretiens de Baton Rouge me viennent à l'esprit, d'abord, des sculptures de Louise Bourgeois. Je pense à ses étranges araignées domestiques qui peuplent les plafonds pour y tisser leurs toiles. Comment la lecture à Paris d'un entretien avec Édouard Glissant, à Baton Rouge, peut-elle m'évoquer une araignée factice et fictive? Peut-être parce que j'avais quitté mes murs pour aller lire les premières pages du livre dans un parc, précisément en face de l'un de ces monstres artistiques façonnés par Bourgeois. A priori, rien ne permet de penser les araignées de Louise Bourgeois et les concepts d'Édouard Glissant dans un même sac. Pourtant, un lien s'est créé, faisant entrer en relation deux expressions poétiques distinctes : dans mon esprit, les pattes d'une araignée géante viennent en cercler les mots, tissant autour d'eux un cocon qui les étouffe. Ma lecture est trouble, je ne parviens pas à saisir le sens de cette pensée.

5 Pour parvenir à laisser respirer les mots, je dois à mon tour délier un à un les fils que cette étrange araignée a tissés autour d'eux. Mais l'araignée est toujours là, obsédante. Elle se métamorphose et devient autre. Désormais, c'est un «babouk » qui arpente les pages de mon livre. Un animal de la famille des aranéides qui n'est pas à proprement parler, dans ma langue, une " araignée ». L'« araignée » est française, parce qu'elle se nomme en langue française; le «babouk» est créole, selon sa dénomination dans ma 
langue maternelle. C'est un heteropoda venatoria, qui vit dans l'île de La Réunion, où je suis né, dans cet espace périphérique amarré par l'histoire par un lien quasi exclusif à la France. Mais mon «babouk» n'habite pas des maisons, il habite des «cases", il ne tisse pas de toiles. Ce n'est décidément pas une araignée du centre: ni d'un centre de toile, et par extension, ni d'un centre de pensée.

6 À Paris, pour retrouver mon «babouk», j'ai donc dû pratiquer un détour : ayant assimilé les mots de Glissant aux sculptures de Bourgeois, j'ai d'abord fait transiter ma conception de l'araignée par une expression poétique et artistique, pour ensuite parvenir à retrouver - et à formuler - mon araignée. Comme cette distinction faite entre ces variétés d'araignées, la pensée vit, se structure et habite le monde selon des modalités différentes. L'une peut habiter l'espace de manière sédentaire, à partir d'un système qu'elle s'est créée; l'autre peut l'habiter de manière mobile, sans système apparent, en se déplaçant constamment. Le «babouk ${ }^{1}$ » erre dans les cases (chacune des cases est un monde), en sautant d'un lieu à un autre et en trainnant sous son abdomen son sac qui lui sert de nid. Ce sac que la mère transporte sous son abdomen est précisément le sac dans lequel j'avais rangé les mots de Glissant et les formes de Bourgeois : toutes mes références culturelles s'y mêlent. Mais une fois les parois de la poche percées, la somme de ces référents se diffracte, ils se mettent à courir et à sauter dans l'espace de ma case, en des trajectoires aussi imprévisibles qu'aléatoires. C'est justement l'effet que ces entretiens provoquent en moi. La pensée d'Édouard Glissant n'est pas une pensée du système, elle n'est pas une pensée du sac, c'est-à-dire de l'Un totalitaire (de l'universel), mais une pensée mobile qui se diffracte et se disperse. Dès lors, je ne peux pas parler de ses mots à lui, sans faire référence aux miens propres parce qu'il parle, à chacun, de son humanité propre : celle d'un lieu singulier dans un temps donné.

7 Il s'agit bien d'un détour multiple. D'abord, parce que pour parler de sa créolité (non pas de sa conception du créole, mais de sa perception du processus de créolisation), Édouard Glissant se place dans un paysage créole autre, celui de la Lousiane ; ensuite parce que, pour le faire parler de sa contemporanéité, Alexandre Leupin l'entraîne dans les sillons du balbutiement des langues et des nations occidentales, dans un autre temps et un autre lieu, ceux du Moyen Âge européen. Le détour est à la fois spatial et temporel : "J'ai appelé ce mouvement la pratique du détour, en particulier chez les peuples de la Caraïbe. Il est étonnant comme les intellectuels de la Caraïbe (je ne parle pas seulement des poètes) se sont impliqués et intéressés aux problèmes soulevés ailleurs, chez d'autres peuples » (106). Par exemple, Édouard Glissant a pratiquement pris en charge une poétique des rives du Mississippi, celle de Faulkner, pour penser et conceptualiser le discours antillais.

8 Cet entretien, qui propose une mise en abyme de l'errance, "n'est pas divagation " (109) : en huit points, il se détourne du lieu commun et des a priori liés à la question de la créolisation, pour mieux y revenir. Le livre se structure dans et par l'errance : il pratique des détours spatiaux et temporels (par l'Europe du Moyen Âge) pour mieux saisir les mouvances contemporaines « des humanités, qui s'acharnent à perdurer, dans leurs diversités, l'inattendu, le mélange, l'inextricable » (153). Il s'appuie sur les errements du passé comme pour aider à mieux prendre pied dans ce qu'Alexandre Leupin a désigné par une « œuvre mangrove » (159) : une œuvre opaque, structurée en un réseau francophone et créolophone complexe, qui fait rhizome avec les langues des différents paysages du monde, qui «renouvelle de fond en comble nos habitudes 
grammaticales » (164). S'agirait-il alors de remuer les fonds d'une vase syntaxique et poétique française, noyée et dépassée par des vents historiques violents qui ont soufflé et dévasté hors des limites du centre?

9 «Ce que je voudrais, avoue Édouard Glissant, c'est qu'on puisse exposer réellement comment, par exemple, les procédés de créolisation linguistique se sont effectués dans les différentes parties de ce qui sera plus tard la France » (69). C'est-à-dire, dans ce qui sera plus tard une France fractionnée et fragmentée par l'expérience de la colonisation, à travers divers lieux de la planète, dont la Caraïbe et l'océan Indien. Et les expériences françaises post-coloniales, interrogées dans les entretiens à partir de la "pensée du système » qui s'est réalisée au Moyen Âge, à partir de cette "poussée de l'universel », de cette "pulsion occidentale", en corrélation avec une pensée transversale de la marge, une "pensée de l'hérésie » (28-29), permettent de nous projeter dans un autre type de rapport et de relation au-dehors des sociétés occidentales.

Il en découle ainsi un point d'interrogation, à la fois consécutif et constitutif de celuici : « est-ce que cette pensée-là [celle née de la "poussée de l'universel"] est encore à même, à elle seule, et dans son système, de nous fournir des ouvertures d'horizon, pour le monde dans lequel nous vivons actuellement? ? (25). Est-ce que cette pensée-là, qui ne reconnaît pas de légitimité à l'existence des diversités, ne risque pas à terme de nous conduire dans une impasse ? Le monde s'ouvre, mais la pensée occidentale héritée des temps médiévaux, si elle ne se dénoue pas, risque de verrouiller nos propres possibilités d'ouverture. Dans ce contexte, « est-ce qu'il n'y a pas une nécessité, étant donné la nature diffractée de ce monde, et l'inextricable de son établissement, de revenir au drame du Moyen Âge, comme nous devrions en revenir aux présocratiques, et aux spiritualités des cultures africaines »? Et, poursuit Édouard Glissant, « de replonger, de savoir comment ça s'est passé entre ces deux portées, de système et de non-système, afin que nous puissions comprendre ce que nous avons à aménager, et comment, dans les relations entre ces deux estimations de l'ordre et du désordre du monde » (25-26) ? La réponse est formulée en une utopie : « on peut [...] concevoir un monde où chacun pourrait être propriétaire sans pour autant chercher à établir la légitimité de l'extension de la propriété » (131). Autrement dit, en faisant retour sur les expériences transversales du passé, et en les détournant du rapport exclusif aux systèmes dominants, on peut parvenir à concevoir d'autres modalités de lecture de l'histoire, et ainsi parvenir à travailler à l'élaboration d'un monde où le principe du monopole d'un système de pensée sur d'autres, qui eux sont transversaux (et non marginaux), ne serait plus valide.

11 Il convient maintenant de revenir sur le parcours d'une pensée qui s'est construite dans le temps à travers des espaces, tant du centre que des périphéries. Cette pensée du centre et de la périphérie, qu'Édouard Glissant développe dans son œuvre depuis La Lézarde (1958), est ici constitutive de son rapport à l'histoire, puisque c'est à partir de mouvements paradoxalement centripètes et centrifuges qu'il s'est créé cette pensée fondatrice (de l'œuvre) et fondamentale (dans l'œuvre) de l'errance. Elle « introduit de nouvelles relations au pays, une opposition poétique aux injonctions de système qui régiraient la fréquentation de celui-ci et qui donc imposeraient une vie altérée du continuum biographique de la communauté » (44). Il n'y a pas de rapport unilatéral à l'histoire, pas plus qu'il n'y a en elle une continuité linéaire. Cette relecture des processus historiques, à partir notamment des concepts abordés dans l'œuvre (créolisation et Relation), permet de retourner le rapport au temps. Non pas un et 
indivisible, mais multiple comme toutes ces sociétés qui ont participé, de près comme de loin, du dedans comme du dehors, à l'élaboration d'un "Chaos monde » (147) - qui, à mon sens, ne peut être un "Tout-Monde ». Il y a juxtaposition des expériences passées aux expériences présentes, qui poussent et retranchent les réticences établies (les choix historiques faits par le passé et posés comme des vérités immuables) pour les rendre à la nature de leurs mouvements : chaotiques dans des jeux de relations transversales, et non pas ordonnés par un système de rapports exclusifs.

Lorsqu'ont lieu ces entretiens, entre 1990 et 1991, Édouard Glissant a déjà publié les deux premiers volumes de sa Poétique : Soleil de la conscience (1956) et L'Intention poétique (1969). Il vient tout juste d'achever son troisième volume, Poétique de la Relation (1990). Le Dis cours antillais (1981) est présent dans son œuvre depuis dix années, et sa production littéraire, tant romanesque que poétique, est déjà riche de La Lézarde (1958), du Sel noir (1960), du Quatrième siècle (1964), de Malemort (1975), de Pays rêvé, pays réel (1985), etc. Les Entretiens de Baton Rouge ne témoigne donc pas d'une pensée en gestation. Le livre vient davantage confirmer les itinéraires empruntés par l'auteur. En outre, il apporte un éclairage sur le fait que l'œuvre dans sa globalité, tout comme l'histoire, ne se structure pas nécessairement de manière linéaire ou selon un ordre chronologique; les entretiens ont lieu au début des années 1990, pourtant ce n'est qu'en 2008, après la publication du Traité du Tout-Monde (1997), de La Cohée du Lamentin (2005) ou d'Une nouvelle région du monde (2006) que le lecteur peut les lire. L'œuvre se structure davantage en un mouvement diachronique dans lequel les mots ne circulent pas (ni cercle, ni spirale) mais se diffractent et se dispersent, arpentant les espaces, parcourant le temps et les langues : «Un écrivain aujourd'hui conjecture son œuvre dans une langue, mais en présence de toutes les langues du monde » (84).

L'œuvre d'Édouard Glissant est un seul et même projet qui s'épand en trajectoires aléatoires au fil des mots et qui tient sa singularité autant dans les fonds sondés par le langage que dans sa structure même: défaire les suites linéaires et lissées, strier les surfaces comme poussent et se développent les racines d'une mangrove. Faire onduler, de rives en rives, les concepts, pour défaire le présupposé universel. Édouard Glissant et Alexandre Leupin ne parlent pas d'une araignée commune perchée sur sa toile, mais d'une araignée poétique et artistique, déjà porteuse de subjectivité, en même temps que d'une araignée singulière, intime, mon fameux «babouk »... Ne pas parler à tous, mais s'adresser à chacun.

\section{NOTES}

1. La graphie du créole réunionnais n'étant pas officiellement codifiée, on pourrait écrire «babouk» autrement, dans une graphie étymologique par exemple, marquant ainsi son lien à la langue française: "babouc» ou "babouque ». Mais mon babouk, araignée dont le genre est masculin, se dit sur l'île et non pas sur le continent. Or selon la graphie choisie (dite KWZ), il ne prend pas de « $\mathrm{s} »$ au pluriel. 


\section{AUTEURS}

\section{STÉPHANE HOARAU}

Maison des Civilisations et de l'Unité Réunionnaise 